\title{
Hexagonal Grid Drawings: Algorithms and Lower Bounds*
}

\author{
Shabnam Aziza and Therese Biedl \\ School of Computer Science, University of Waterloo, \\ Waterloo, Ontario N2L 3G1, Canada \\ $\{$ saziza, biedl\}@uwaterloo.ca
}

\begin{abstract}
We study drawings of graphs of maximum degree six on the hexagonal (triangular) grid, with the main focus of keeping the number of bends small. We give algorithms that achieve $3.5 n+3.5$ bends for all simple graphs. We also prove optimal lower bounds on the number of bends for $K_{7}$, and give asymptotic lower bounds for graph classes of varying connectivity.
\end{abstract}

\section{Introduction}

There are numerous algorithms to draw 4-graphs (graphs of maximum degree of at most four) on the 2D rectangular (orthogonal) grid [3, 8-11]. All 4-graphs can be drawn with at most $2 n+2$ bends [3], and there are arbitrarily large graphs that need $\frac{11}{6} n$ bends [1]. In 3D, orthogonal drawings exist for all 6 -graphs [4, 13-15]. In this paper, we study hexagonal drawings, which are embeddings of 6 -graphs in the 2D hexagonal grid. We consider the hexagonal grid to consist of horizontal gridlines (rows), vertical gridlines (columns) and diagonals; this is the same grid as the "standard" hexagonal grid (with $60^{\circ}$ angles) after a shear in the $x$-direction. See also Fig. 1.

Only few results are known for hexagonal drawings. The algorithm by Tamassia [10] to obtain bend-minimum orthogonal drawings of planar graphs can be extended to the hexagonal grid as well. Kant [6] showed how to draw 3-connected

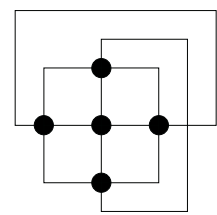

(a)

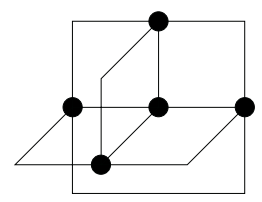

(b)

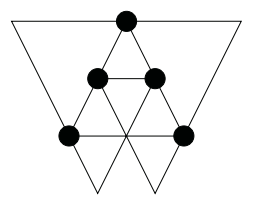

(c)

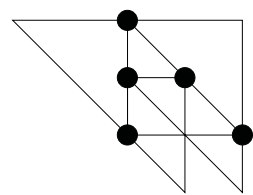

(d)

Fig. 1. Different types of grid drawings of $K_{5}$ : (a) 2D orthogonal, (b) 3D orthogonal, (c) hexagonal with $60^{\circ}$ angles, (d) hexagonal with diagonals.

\footnotetext{
* Research supported by NSERC. These results appeared as part of the MMath thesis of the first author at University of Waterloo.
} 
cubic planar graphs on the hexagonal grid. Tollis [12] uses a similar grid called tri-hexagonal grid for wiring VLSI layouts.

In this paper, we provide an algorithm that draws all graphs with maximum degree 6 on the hexagonal grid, and achieves $3.5 n+3.5$ bends. We also study lower bounds on the number of bends for $K_{7}$ and for larger graphs.

\section{Algorithms}

Our algorithms are inspired by the algorithm of Biedl and Kant [3] for drawing biconnected 4-graphs on the orthogonal grid. They use a vertex ordering known as st-ordering, which is an ordering $v_{1}, v_{2}, \ldots, v_{n}$ of the vertices such that each $v_{i}, 2 \leq i \leq n-1$, has at least one predecessor, i.e., a neighbour $v_{h}$ with $h<i$ and at least one successor, i.e., a neighbour $v_{j}$ with $j>i$. The edges from $v_{i}$ to its predecessors [successors] are called incoming [outgoing] edges of $v_{i}$. The number of incoming [outgoing] edges of vertex $v_{i}$ are denoted by $\operatorname{indeg}\left(v_{i}\right)\left[\operatorname{outdeg}\left(v_{i}\right)\right]$. For any biconnected graph, and any two vertices $s, t$, an $s t$-ordering exists with $v_{1}=s$ and $v_{n}=t[7]$ and can be computed in linear time [5].

Assume from now on that $G$ is a biconnected 6-graph without loops, and $v_{1}, \ldots, v_{n}$ is an $s t$-ordering of $G$. Let $G_{j}$ be the graph induced by $v_{1}, \ldots, v_{j}$. An edge $\left(v_{i}, v_{k}\right)$ with $i \leq j<k$ is called an unfinished edge of $G_{j}$. For $j=1, \ldots, n$, we create a drawing of $G_{j}$ such that every unfinished edge ends in a free ray, i.e., every unfinished edge is drawn up to a point, and there exists a ray (along a grid line) from this point that does not contain any vertex or edge segment in it, and is the free ray for only one unfinished edge. These rays must go in direction north $(\mathrm{N})$, north-west (NW) or west (W). Fig. 2 shows a suitable drawing of $G_{1}$ and illustrates the invariant.
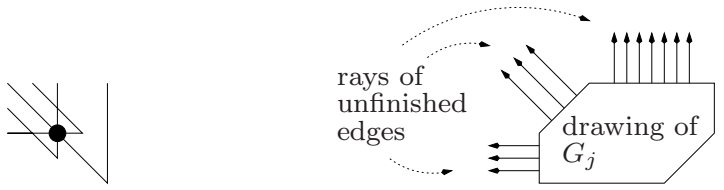

Fig. 2. Embedding of the first vertex, and the maintained invariant.

Now assume that we have a suitable drawing of $G_{j-1}$. If $\left(v_{i}, v_{j}\right)$ is an incoming edge of $v_{j}$, then there is an unfinished edge at $v_{i}$, and hence a ray associated with it. (We choose one arbitrarily if there is more than one ray.) We add (if needed) bends in these rays, and new line segments in new grid lines that are fully outside the drawing of $G_{j-1}$ in such a way that all incoming edges meet in one grid point at which we place $v_{j}$. Then we assign rays to outgoing edges of $v_{j}$, adding more bends (if needed) to enforce that rays go into one of the three allowed directions. The specific drawing of $v_{j}$ depends on the number and directions of rays of incoming edges of $v_{j}$. There are many cases here; Fig. 3 shows some of them.

Studying all cases yields that each vertex $v \neq v_{1}, v_{n}$ needs at most indeg $(v)+1$ bends, which leads to a bound of $m+n+O(1) \leq 4 n+O(1)$. But this is not 

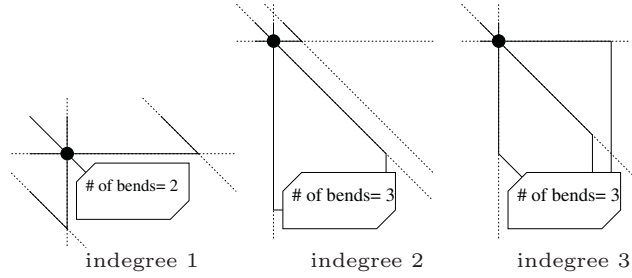

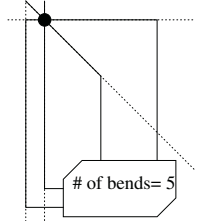

indegree 4

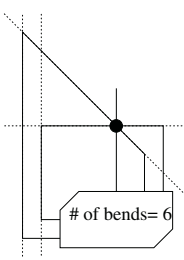

indegree 5

Fig. 3. Some of the cases of embedding vertices. Dotted lines indicate new grid lines.

tight. To obtain a better bound on the number of bends, we developed a potential function argument, which to our knowledge is a new idea in graph drawing. We assign potential $p_{d}, d \in\{N, N W, W\}$ to each unfinished edge whose free ray ends in direction $d$. Let $\phi(j)$ be the sum of the potentials of the drawing of $G_{j}$. Then the amortized cost of a vertex $v_{j}, 1 \leq j \leq n$, is,

$$
\hat{c}\left(v_{j}\right)=\text { number of bends added when placing vertex } v_{j}+\phi(j)-\phi(j-1) \text {. }
$$

Hence the total number of bends is $\sum_{v \in V} \hat{c}(v)$.

Theorem 1. Any biconnected 6-graph without loops has a hexagonal grid drawing with at most $3.5 n+3.5$ bends.

Proof. We choose as potentials $p_{N}=p_{W}=\frac{5}{8}$ and $p_{N W}=\frac{1}{8}$. The amortized cost at $v_{i}$ is determined uniquely from the number of bends needed when placing $v_{i}$ and the directions of the incoming and outgoing edges. Let $\hat{c}_{i}$ be the maximum amortized cost of a vertex with indegree $i$. Going through the cases, one obtains $\hat{c_{0}}=5.25, \hat{c_{1}}=3.5, \hat{c_{2}}=3.25, \hat{c_{3}}=3, \hat{c_{4}}=3.25, \hat{c_{5}}=3.5$ and $\hat{c_{6}}=5.25$. (The cases in Fig. 3 are some of those where these bounds are tight.) So the number of bends is

$$
\sum_{v \in V} \hat{c}(v) \leq \sum_{i=0}^{6} \hat{c_{i}} n_{i}=5.25 n_{0}+3.5 n_{1}+3.25 n_{2}+3 n_{3}+3.25 n_{4}+3.5 n_{5}+5.25 n_{6} .
$$

Since $n_{0}=1$ and $n_{6} \leq 1$ for an st-ordering of a 6 -graph, this yields the desired bound.

Our algorithm can be expanded with similar techniques as in [3] to handle graphs that are not biconnected or that have loops. The proofs of the following theorems are omitted.

Theorem 2. Any connected 6-graph without loops with $n \geq 3$ can be drawn on the hexagonal grid with at most $4.2 n$ bends.

Theorem 3. Every simple connected 6-graph can be drawn on the hexagonal grid with at most $3.5 n+3.5$ bends.

Theorem 4. Any biconnected 6-graph can be drawn on the hexagonal grid with at most $3.5 n+3.5+\frac{1}{4} \ell$ bends, where $\ell$ is the number of loops in the graph. 
The area of our construction may be exponential, since in some cases we add $O(w)$ new rows to a drawing of width $w$, or $O(h)$ many columns to a drawing of height $h$. The area can be reduced to quadratic if we allow more bends.

Theorem 5. Every biconnected 6-graph without loops can be drawn on the hexagonal grid with at most $6 n+2$ bends and area $O\left(n^{2}\right)$.

\section{Lower Bounds}

We now turn to lower bounds on the number of bends of hexagonal grid drawings. We start with $K_{7}$, the complete graph on 7 vertices. This graph requires 20 bends in a 3D orthogonal layout [14]. In the hexagonal grid, it can be drawn with 18 bends (see Fig. 4), and as we show now, this bound is tight. So assume that an arbitrary drawing of $K_{7}$ is fixed. Let $r$ be the number of rows that are truly used, i.e., they contain either a vertex or a segment of an edge. Similarly let $c$ and $d$ be the number of truly used columns and diagonals. We first show $r+c+d \geq 18$, for which by symmetry it suffices to show $r+d \geq 12$.

Lemma 1. In any hexagonal drawing of $K_{7}, r+d \geq 12$.

Proof. We use a cut-argument similar as in [1]. A (vertical) cut is a vertical line that does not coincide with a column. An $(x, y)$-cut is a cut with $x$ vertices on one side and $y$ vertices on the other. The edges between vertices on different sides are called cut-edges. Each cut-edge has at least one segment crossed by the cut, and hence truly uses a grid line that crosses the cut (i.e., a row or a diagonal). A $(3,4)$-cut in $K_{7}$ has 12 cut-edges, and hence immediately implies that $r+d \geq 12$. However, such a cut need not always exist. We distinguish cases.

Assume there is a column $c_{6}$ with exactly six vertices in it. We consider two cuts; one cut $c_{l}$ immediately to the left of $c_{6}$ and one cut $c_{r}$ immediately to the right of $c_{6}$. We assume that the seventh vertex is to the left of $c_{6}$, so $c_{l}$ has 6 cut-edges. Let $(u, v)$ be an edge for which $u, v$ are both in $c_{6}$, but not consecutive in $c_{6}$. Then $(u, v)$ cannot be drawn as a straight line. We call $(u, v)$ a non-straight edge and note that there are $\left(\begin{array}{l}6 \\ 2\end{array}\right)-5=10$ non-straight edges. See also Fig. 4.

The drawing of each non-straight edge thus must leave column $c_{6}$ and then return to it. Say it leaves towards the left side, then it crosses the cut $c_{l}$ when it leaves, and crosses the cut $c_{l}$ again when it returns. If $a$ non-straight edges go
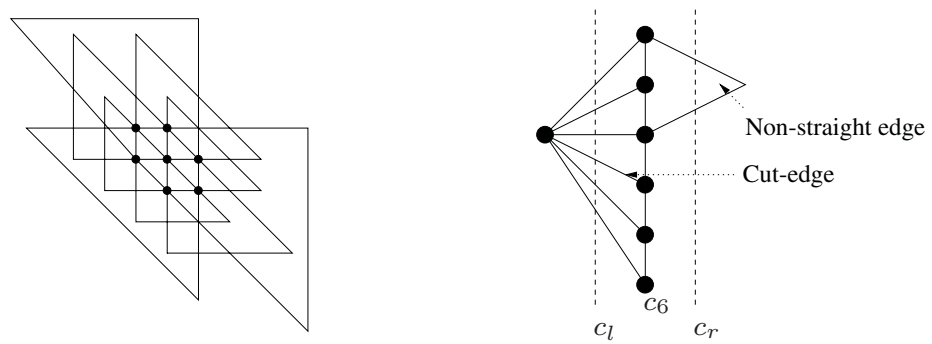

Fig. 4. $K_{7}$ drawn with 18 bends, and the case with 6 vertices in the same column. 
left, then there are therefore at least $2 a$ edge segments that cross cut $c_{l}$ and truly use a row or a diagonal. Since $c_{l}$ also has 6 cut-edges, we have $r+d \geq 2 a+6$.

The other $10-a$ non-straight edges go right and cross $c_{r}$ twice, so $r+$ $d \geq 2(10-a)$. Altogether therefore $r+d \geq \max \{2 a+6,2(10-a)\}$. Since the value of $a$ is unknown, we take the minimum over all possible values, and get $r+d \geq \min _{0 \leq a \leq 10} \max \{2 a+6,2(10-a)\}=14$.

All other cases are treated similarly: we count how many cut-edges are on $c_{l}$ and $c_{r}$ and how many non-straight edges there are, and apply the formula to compute the lower bound on $r+d$. In all cases, we get $r+d \geq 12$. For space reasons, we omit the details of these cases, but list the cases so the reader can verify that all cases have been covered.

- A column $c$ contains 7 vertices.

- A column $c$ contains 5 vertices, and there are 2 vertices on one side of $c$.

- A column $c$ contains 5 vertices, and there is 1 vertex on each side of $c$.

- A column $c$ contains 4 vertices, and there are 3 vertices on one side of $c$. (In this case $c_{l}$ or $c_{r}$ is a $(3,4)$-cut.)

- A column $c$ contains 4 vertices, and there are 1 or 2 vertices on one side of $c$.

- A column $c$ contains 3 vertices, and there are 3 or 4 vertices on one side of $c$. (In this case $c_{l}$ or $c_{r}$ is a $(3,4)$-cut.)

- A column $c$ contains 3 vertices, and there are 2 vertices on each side of $c$.

- All columns contain at most 2 vertices. Applying a scan from left to right, one can show that then there must exist a $(3,4)$-cut.

We can relate $r+c+d$ to the number of bends, similarly as in [2].

Lemma 2. In a hexagonal drawing of a graph with $n$ vertices and $m$ edges, let $b$ be the number of bends and let $r, c, d$ be the number of truly used rows, columns and diagonals. Then $b \geq r+c+d-3 n+m$.

Combining this with $r+c+d \geq 18$ give the lower bounds for $K_{7}$.

Theorem 6. Any hexagonal drawing of $K_{7}$ has at least 18 bends.

Using $K_{7}$ and other small graphs, we can build arbitrarily large graphs that also have a large lower bound on the number of bends, similarly as done in [1] for orthogonal 2D drawings and in [14] for orthogonal 3D drawings. We give the detailed construction for one (illustrative) case.

Theorem 7. For any $n$, there is a 3-connected simple graph on $n^{\prime}>n$ vertices that requires at least $1.87 n^{\prime}$ bends.

Proof. The graph for this lower bound is illustrated in the bottom left entry of Table 1. We take an even number $c$ of copies of $K_{7}$ and place half of them in the first row and half of them in the second row. In each copy of $K_{7}$, we subdivide one edge; then we identify the subdivision vertex in the first row with the one in the second row. Also, in each copy we delete an edge (not incident to the subdivision vertex $)$. Then we add an edge between the $i$ th and the $\left(\left(i \bmod \frac{c}{2}\right)+1\right)$ st copy of $K_{7}$ in each row. 
Table 1. Lower bounds for arbitrarily large graphs.

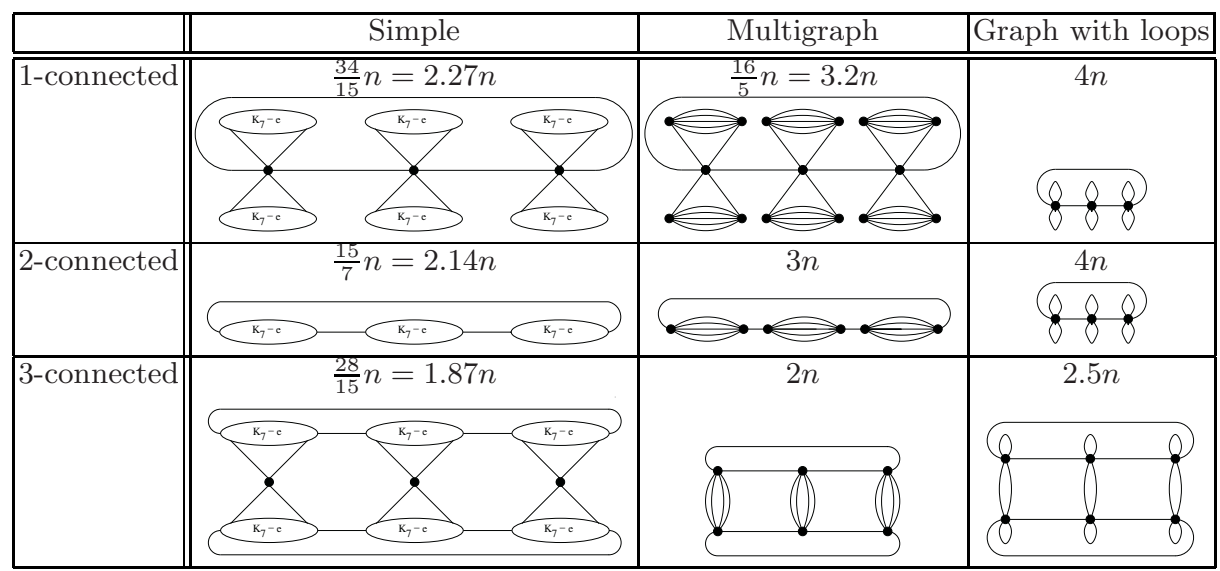

Recall that $K_{7}$ needs 18 bends. Subdividing an edge lowers this to 17 bends, because the subdivision vertex could take the place of a bend. Deleting an edge lowers this to 14 bends since one can show that in any hexagonal drawing, we can add an edge while adding at most 3 bends. Thus each copy needs at least 14 bends, so in total we need at least $28 c$ bends for $n=15 c$ vertices, and the total number of bends is $\frac{28 n}{15}=1.87 n$.

Similar (and often easier) constructions can be used to obtain lower bounds for various types of graphs and connectivity; see Table 1.

\section{References}

1. T. Biedl. New lower bounds for orthogonal drawings. J. Graph Algorithms Appl., 2(7):1-31, 1998.

2. T. Biedl. Relating bends and size in orthogonal graph drawings. Inform. Process. Lett., 65(2):111-115, 1998.

3. T. Biedl and G. Kant. A better heuristic for orthogonal graph drawings. Comput. Geom., 9(3):159-180, 1998.

4. P. Eades, A. Symvonis, and S. Whitesides. Three-dimensional orthogonal graph drawing algorithms. Discrete Appl. Math., 103(1-3):55-87, 2000.

5. S. Even and R. E. Tarjan. Computing an st-numbering. Th. Comput. Sci., 2:339344, 1976.

6. G. Kant. Hexagonal grid drawings. In Graph-theoretic Concepts in Computer Science, vol. 657 of Lect. Notes in Comput. Sci., pages 263-276. Springer, 1993.

7. A. Lempel, S. Even, and I. Cederbaum. An algorithm for planarity testing of graphs. In Theory of Graphs (Internat. Sympos., Rome, 1966), pages 215-232. New York, 1967.

8. A. Papakostas and I. G. Tollis. Algorithms for area-efficient orthogonal drawings. Comput. Geom., 9(1-2):83-110, 1998.

9. J. Storer. On minimal-node-cost planar embeddings. Networks, 14(2):181-212, 1984. 
10. R. Tamassia. On embedding a graph in the grid with the minimum number of bends. SIAM J. Comput., 16(3):421-444, 1987.

11. R. Tamassia and I. G. Tollis. Planar grid embedding in linear time. IEEE Transactions on Circuits and Systems, 36(9):1230-1234, 1989.

12. I. Tollis. Wiring layouts in the tri-hexagonal grid. Constraints, 3(1):87-120, 1998.

13. D. R. Wood. An algorithm for three-dimensional orthogonal graph drawing. In Graph Drawing, volume 1547 of Lecture Notes in Comput. Sci., pages 332-346. Springer, 1998.

14. D. R. Wood. Lower bounds for the number of bends in three-dimensional orthogonal graph drawings. J. Graph Algorithms Appl., 7(1):33-77, 2003.

15. D.R. Wood. Optimal three-dimensional orthogonal graph drawing in the general position model. Th. Comput. Sci., 299(1-3):151-178, 2003. 